\title{
FUENTES DE LA DESIGUALDAD ECONÓMICA EN ECUADOR
}

\section{SOURCES OF ECONOMIC INEQUALITY IN ECUADOR}

Recibido: 30/06/2016

Aceptado: $\mathrm{D} / 10 / 2016$

\author{
José-Ramírez Álvarez ${ }^{1}$ \\ jose.ramirez@epn.edu.ec \\ Juan Díaz Sanchez ${ }^{2}$ \\ juan.diaz@epn.edu.ec
}

\section{Forma sugerida de citar:}

Ramírez, J., y Díaz, J. (2017). Fuentes de la desigualdad económica en Ecuador. Revista Economía y Política, XIII (25), páginas.9-28. doi: 10.25097/rep.n25.2017.01

\section{Resumen}

En las últimas décadas, el problema de la desigualdad económica ha tomado relevancia en la mayoría de economías en el mundo. Ecuador no ha sido la excepción. En el año 2014, el 5\% de la población más rica del Ecuador concentró 46 veces el ingreso del 5\% de la población más pobre. Esta disparidad entra en seria controversia con los principios de desarrollo estipulados en la constitución ecuatoriana. En este contexto, el presente documento analiza las principales fuentes de ingresos que contribuyen a la desigualdad económica del Ecuador. Los datos utilizados provienen de la Encuesta de Condiciones de Vida 2013-2014.

Palabras Claves: ingreso, desigualdad, Ecuador.

\section{Abstract}

In the last decades, the economic inequality problem has taken relevance in most economies in the world. Ecuador has been no exception. In 2014 , the $5 \%$ of the richest population of Ecuador concentrated 46 times the income of $5 \%$ of the poorest population. This disparity creates a serious controversy with the development principles stipulated in the ecuadorian constitution. In this context, this paper analyzes the main income sources that contributes the economic inequality in Ecuador. The data come from the Survey of Living Conditions 2013-2014.

Keywords: income, inequality, Ecuador.

Código DOI: http://dx.doi.org/10.25097/rep.n25.2017.01

Código JEL: D63, D31

Profesor. Escuela Politécnica Nacional, Facultad de Ciencias, Quito, Ecuador.

2 Profesor. Escuela Politécnica Nacional, Facultad de Ciencias, Quito, Ecuador. 


\section{Introducción}

Uno de las teorías más importantes y controversiales en el marco de desarrollo constituye la paradoja de Kuznets (1955), o lo que se conoce como la hipótesis de la "U invertida". Esta hipótesis sostiene que la desigualdad crece en las primeras etapas de desarrollo, producto del proceso de industrialización que tiende a concentrar la distribución de recursos económicos. Esta tendencia se atenúa en fases de desarrollo más avanzadas a causa de la inversión gradual de capital humano, al punto de revertirse y tomar niveles de distribución más igualitarios; esto independientemente de la política pública y choques económicos ${ }^{3}$.

Este planteamiento parece explicar la dinámica distributiva en el mundo. De acuerdo a estadísticas de Milanovic (2011) la desigualdad ha crecido desde inicios del siglo XIX hasta mediados del siglo XX (Figura 1); época principalmente caracterizada por la modernización. No obstante, a partir del año 1980 esta tendencia aparentemente se pierde en lo que se podría decir la cima de la " $U$ invertida", sin una notable mejora en términos de equidad pese al considerable incremento del PIB per cápita mundial.

Figura 1. Desigualdad económica en el mundo.

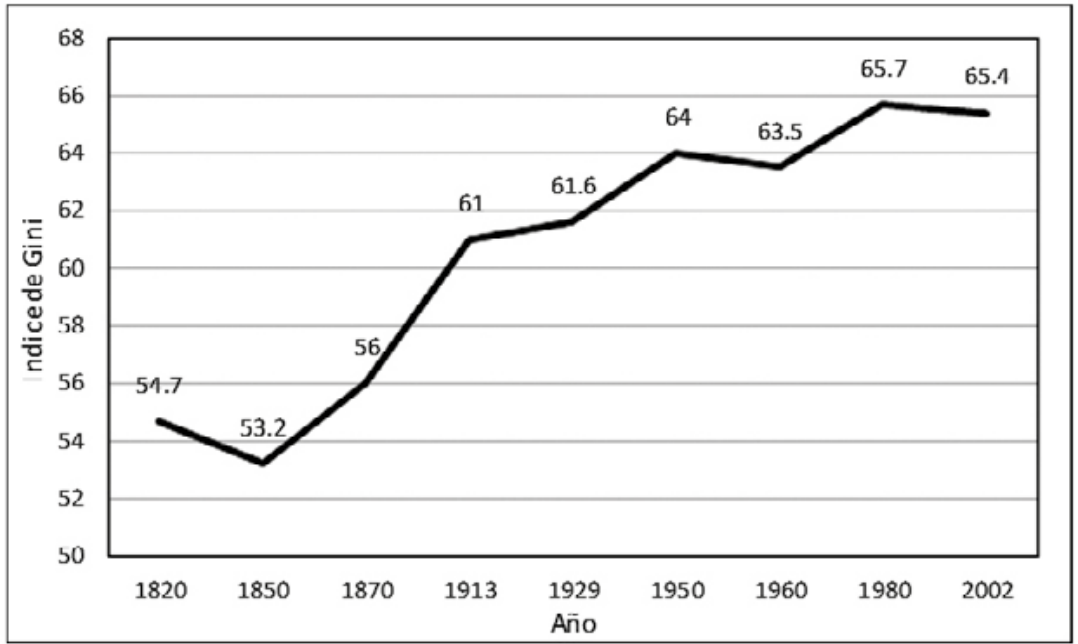

Fuente: Milanovic (2011)

3 De acuerdo a Pikety (2014), el trabajo de Kuznetz es uno de los estudios de mayor importancia dado el levantamiento de datos y la construcción de la primera serie histórica de la desigualdad económica en el siglo XIX. No obstante, su hipótesis de la U invertida tiene argumentos teóricos y empíricos frágiles, pues gran parte de la reducción de la desigualdad en esa época según Pikety, se debió a las guerras mundiales, las crisis económicas y las políticas públicas que éstas conllevaban. 
Este tipo de desarrollo pasivo se torna severo cuando se observa las desigualdades en la distribución del ingreso mundial. De acuerdo a estadísticas de Dikhanov (2005), el $20 \%$ de la población más rica concentra el $75 \%$ del ingreso mundial, mientras que el $20 \%$ de la población más pobre concentra solo $1.5 \%$ de este ingreso.

En Ecuador, la realidad económica no parece estar lejos de lo que acontece en el mundo. Según estadísticas de la Encuesta Condiciones de Vida al año 2014 (Tabla 1 ), el $5 \%$ de la población más rica concentra el $23.5 \%$ del ingreso total mientras que el $5 \%$ de la población más pobre concentra solo el $0.5 \%$; es decir, existe una polarización entre ambos estratos de 45.98 veces.

Tabla 1. Distribución del Ingreso en Ecuador. Año 2014

\begin{tabular}{lc|ccc}
\hline \multicolumn{3}{c}{ Acumulación del ingreso total } & & Número de \\
veces
\end{tabular}

Fuente: Encuesta de Condiciones de Vida. Año 2013-2014

Esta situación es aún más grave cuando se evidencia que el crecimiento económico de los últimos 15 años no ha estado acompañado de mejoras significativas en la distribución del ingreso (Figura 2). En promedio, el PIB percápita creció $9.9 \%$ en el periodo $200-2015$, mientras el coeficiente de Gini sobre el ingreso bruto disminuyó en tan solo $1.1 \%$ en el mismo periodo.

Este fenómeno podría advertir un posible acercamiento a la cima de la "u invertida" de Kuznets donde no se logren mayores reducciones en la desigualdad económica, y por ende sea estructuralmente difícil mejorar la distribución del ingreso mediante política pública.

El problema de la desigualdad económica es elemental en Ecuador dado el alineamiento político de las distintas legislaciones con los principios de desarrollo. En particular, el artículo 276 de la Nueva Constitución del Ecuador establece, entre varios otros objetivos, que el régimen de desarrollo debe sumarse a la causa de "Construir un sistema económico, justo, democrático, productivo, solidario y sostenible basado en la distribución igualitaria de los beneficios del desarrollo, de los medios de producción y en la generación de trabajo digno y estable". Así también, el primer objetivo del Plan Nacional del Buen Vivir establece propósito "Garantizar una vida digna con iguales oportunidades para todas las personas". 
Figura 2. Coeficiente de Gini por ingreso y PIB per-cápita en Ecuador. Periodo 2000-20154

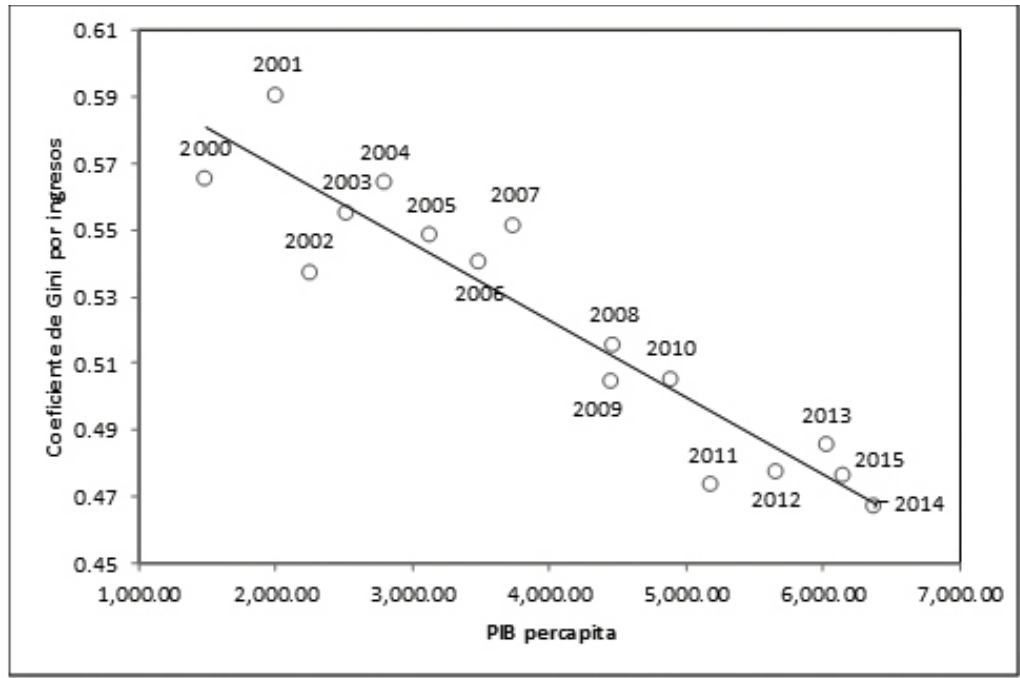

Fuente: Coeficiente de Gini: Sistema de Indicadores Sociales del Ecuador. PIB-percapita: Cuentas Nacionales del Banco Central del Ecuador

En este contexto, el presente documento analiza las principales fuentes de ingresos que determinan la desigualdad económica en Ecuador. Para ello, se emplea la curva de Lorenz y el coeficiente de Gini, así como también el análisis marginal de la desigualdad planteado por Lerman y Yitzaki (1985). La información utilizada para este estudio proviene de los ingresos por salarios, ganancias, retornos de capital y transferencias registradas en la Encuesta de Condiciones de Vida del año 2013-2014.

Este tipo de análisis es importante en la práctica pues ayuda a entender el comportamiento que adquiere la desigualdad económica cuando varían sus fuentes de ingreso, y elaborar con ello alternativas de política pública que permitan disminuir la concentración de la riqueza de manera efectiva y permanente.

Existen varios trabajos en la literatura que han analizado el comportamiento de la desigualdad económica por fuente de ingreso. En América Latina, Wodon et al.

4 Los altos niveles de desigualdad y pobreza a comienzos de siglo fueron consecuencia de la fragilidad del aparato productivo y las instituciones estatales causada por el fenómeno del niño de 1998 y la crisis bancaria de 1999. Luego, la estabilidad nominal provista por la dolarización permitió que la distribución del ingreso mejorara paulatinamente hasta el 2006, alcanzando características similares a la distribución del año 1995 (Ramírez, 2008). Pese a la crisis de los suprime en 2008 y al descenso del precio del barril de petróleo en 2008 y 2015 , los niveles de desigualdad continuaron disminuyendo como efecto del fortalecimiento de los programas sociales y los servicios públicos a partir de la nueva constitución y la ejecución de una agenda de desarrollo. 
(2000), Wodon y Yitzhaki (2002a), Wodon y Yitzhaki (2002b), Medina y Galvan (2005) son algunos ejemplos que ilustran la descomposición de la desigualdad por fuentes de ingreso utilizando datos de la encuesta de laborales y de condiciones de vida.

En Ecuador, existen estudios importantes al respecto. Ramírez (2008) analiza la desigualdad económica empleando datos de la Encuesta de Condiciones de Vida 2005-2006 y encuentra que fuentes de ingreso como los salarios, las ganancias e ingresos de capital contribuyen fuertemente a la desigualdad económica en Ecuador. Asimismo, encuentra que las remesas, el bono de desarrollo y algunos programas de salud tiene un efecto positivo sobre la distribución de ingresos. En la misma línea, Ponce y Vos (2014) examinan el comportamiento de la desigualdad económica en Ecuador en el periodo 1990 a 2010 utilizando la Encuesta Nacional de Empleo, Desempleo y Subempleo. Estos autores muestran que los ingresos de capital y las remesas tienen una relación directamente proporcional con la desigualdad económica, aunque en el caso de las remesas esta relación tiende a desaparecer en el año 2010 especialmente para los hogares urbanos. Por el contrario, encuentran que los ingresos laborales y las transferencias de dinero contribuyen positivamente a una mejor distribución del ingreso, sin embargo, para los ingresos laborales este efecto disminuye paulatinamente en el tiempo hasta volverse insignificativo.

El documento se encuentra estructurado de la siguiente manera: la segunda sección expone algunos antecedentes de la teoría del desarrollo y la desigualdad. La tercera sección describe la curva de Lorenz, el índice de Gini y la elasticidad de Gini por fuente de ingreso. La quinta sección expone los resultados obtenidos; y finalmente, la sexta sección realiza las conclusiones del trabajo, junto con algunas recomendaciones en política económica para disminuir la desigualdad económica en Ecuador.

\section{Marco Teórico}

\subsection{La Teoría del desarrollo}

La teoría de desarrollo constituye uno de los campos de mayor discusión en cuanto al progreso económico y social de las naciones. Esta teoría nace durante la transición del siglo XVIII y siglo XIX, apoyándose en el pensamiento clásico para dar cabida a la modernización y la revolución industrial en el progreso de las naciones. A mediados del siglo XIX, esta confluencia filosófica desembocó en la idea de que el desarrollo constituye "la capacidad de satisfacer las necesidades humanas mediante la innovación y el incremento de la producción". 
A inicios del siglo XX, esta corriente productivista incluyó el enfoque cuantitativo de Pigou al suponer la interdependencia del bienestar y la renta nacional, y la posibilidad de medir el desarrollo mediante la contabilidad nacional. Este criterio fue adoptado por la línea keynesiana, al entender el desarrollo plenamente como el incremento de la capacidad productiva o el crecimiento del PIB per cápita. Este lineamiento fue el sustento de varios argumentos que propugnaban una mayor tasa de ahorro e inversión a favor del desarrollo (Satrustegui, 2009).

Desde entonces han surgido varios debates sobre la naturaleza misma de los procesos de desarrollo, con el objeto de dar satisfacción a los diversos principios vinculados con el bienestar humano. Algunos de estos debates, por ejemplo, han girado en torno a lo que se conoce como las teorías de desarrollo alternativo, las cuales amplían la concepción tradicional del desarrollado incluyendo temas como: la desigualdad del ingreso y la pobreza, el género, el irrespeto a la libertad y a los derechos humanos, el progresivo deterioro del ambiente, el desarrollo comunitario, entre otros (Veltmeyer, 2001; Hettne, 1995).

La escuela del desarrollo alternativo sentó las bases para impulsar una redefinición del desarrollo como tal, a partir de un nuevo paradigma de bienestar. Sobresale aquí el enfoque capacidades de Amartya Sen. Este nuevo enfoque es el centro de varias iniciativas para promover el desarrollo mediante el incremento de las oportunidades de las personas.

Una de las instituciones que se basa en este enfoque es el Programa de la Naciones Unidades para el Desarrollo (PNUD), la cual define el desarrollo como: "El proceso mediante el cual se busca la ampliación de las oportunidades de las personas, aumentando sus derechos y capacidades".

Figura 3. Desarrollo Humano de acuerdo a la PNUD

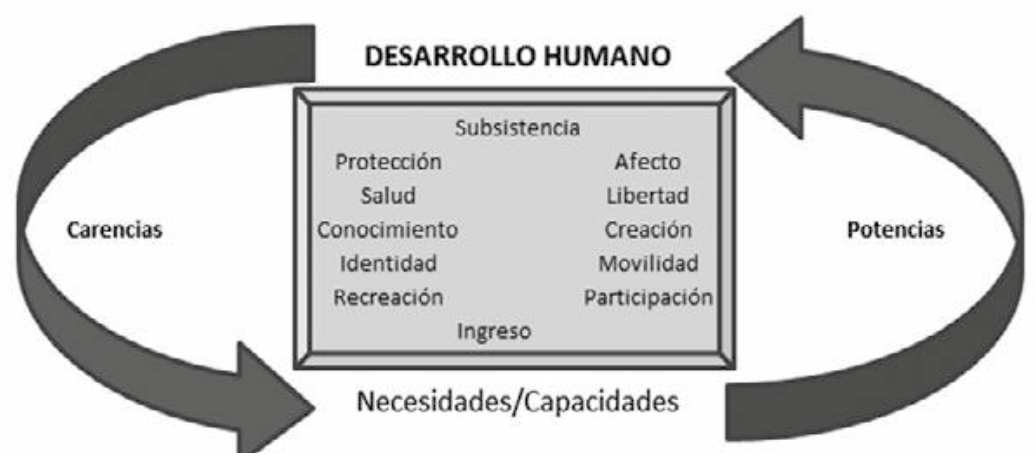

Fuente: Programa de las Naciones Unidades para el Desarrollo (2002) 
Es decir, el proceso de desarrollo consiste no solo en garantizar el acceso por igual a una serie de bienes y servicios básicos (salud, educación, estado de derecho y libertad, participación, seguridad, etc), sino también en potenciar las distintas capacidades de las personas para vivir la vida que ellas deseen.

\section{2 ¿Desigualdad económica o social?}

Un primer significado de la desigualdad en términos monetarios es dado por Kuznets (1953) en cita de Núñez (2006), al establecer que "cuando hablamos de 'desigualdad de la renta', simplemente nos referimos a las diferencias de renta, sin tener en cuenta su deseabilidad como sistema de recompensas o su indeseabilidad como esquema que contradice cierta idea de igualdad". Pese a que esta definición es sencilla y una de las más utilizadas en los análisis de incidencia distributiva por su carácter cuantitativo, deja de lado otras características sociales, pues su interés central es el ingreso.

En la teoría de justicia distributiva, Rawls (1971) plantea que la equidad debe comprender la asignación equilibrada de un conjunto de "bienes primarios". Estos bienes constituyen "todo lo que un individuo racional presume querer, incluyendo derechos, libertades y oportunidades, ingreso y riqueza”. Según Rawls, la distribución de estos bienes debe estar fundamentada en la preponderancia de la libertad y en la igualdad de condiciones sobre todo en estratos marginados.

En un marco más normativo, Atkinson (1975) establece que la desigualdad debe ser definida partiendo del hecho que la igualdad es "deseable per se". En este sentido, Atkinson manifiesta en cita de Coral de Rio (2001) que " $L a$ mera existencia de disparidades de renta y riqueza no constituye una base suficiente para realizar afirmaciones sobre la justicia e injusticia; es necesario determinar que los individuos implicados sean comparables en lo que se refiere a otros aspectos relevantes". Este planteamiento señala la importancia de relativizar mediante algún criterio social, la posición de los individuos antes de cualquier análisis de desigualdad en el ingreso, ya que puede producirse un sesgo sustancial en los resultados si se obvia las diversas circunstancias y factores sociales (edad, género, actividad, carga familiar, etc.) que marcan la distribución de la renta.

En suma a estas acepciones, existe la rigurosa definición dada por Sen (1979). En ella, se establece que la desigualdad social debe concebir una realidad más amplia y diversa que la establecida en los enfoques tradicionales, añadiendo una dimensión que capture las "capacidades básicas" (la habilidad de convertir los bienes en lo que realmente importa, ya sea la libertad de hacer o ser lo que 
se desee) y la urgencia de las mismas como aspectos moralmente relevantes dentro del análisis. Según Sen, la igualdad de las capacidades básicas puede ser vista como "una extensión natural del enfoque de Rawls, cambiando la atención de lo que son los bienes propiamente, a lo que los bienes producen en los seres humanos, ... ,[es decir] en una dirección no fetichista".

Todos estos enfoques poseen un elemento analítico común que le provee una mirada económica al fenómeno de desigualdad: el ingreso o riqueza de los individuos. En rasgos muy generales, la desigualdad en términos económicos se centra en capturar y/o sintetizar las disparidades en una variable focal, en este caso ingreso o riqueza, sobre un conjunto finito de individuos. Las diferencias en esta variable expresan de manera directa o indirecta las diferencias en el bienestar de los individuos como síntoma de una ventaja y/o desventaja personal con respecto a los demás.

Según Sen y Foster (2005), la medición de la desigualdad económica se puede dividir en dos categorías. Por un lado, existe la medición objetiva o positivista que emplea instrumentos de la estadística para cuantificar la variación relativa del ingreso, sin explicitar ningún juicio de valor. Por otro lado, existe la medición normativa, la cual conceptualiza la medición de la desigualdad económica en congruencia con principios de la teoría del bienestar.

\section{Metodología}

\subsection{Curva de Lorenz y Coeficiente de Gini}

La Curva de Lorenz $L_{Y}(p)$ constituye uno de los instrumentos gráficos más utilizados para visualizar la distribución de una variable (generalmente el ingreso) y observar su variación entorno al orden de la población. Esta curva traza el valor esperado acumulado y normalizado de una variable focal $Y$, para cada cuantil de la población.

En el caso que estos cuantiles sean calculados tomando como referencia la distribución de otra variable $X$, la curva de Lorenz se conoce como curva de concentración $C_{X}^{Y}(p)$.

Pese a su complicada formulación matemática, la curva de Lorenz permite dar valiosas interpretaciones sobre la distribución de un variable focal. En particular, para un cuantil cualquiera $p_{1}$, se puede señalar que el $p_{1} \%$ de la población más pobre acumula el $L_{Y}\left(p_{1}\right) \%$ de la variable $Y$; o lo que es lo mismo, el $\left(1-p_{1}\right) \%$ de la población más rica acumula el $\left(1-L_{Y}\left(p_{1}\right)\right) \%$ de la variable $Y$ (Figura 4$)$. 
En el caso de una curva de concentración $C_{X}^{Y}(p)$, esta interpretación también es válida, no obstante se debe recordar que el cuantil $p_{1}$ es calculado tomando como referencia la distribución de la variable focal $X$.

Figura 4. Curva de Lorenz y Curva de Concentración
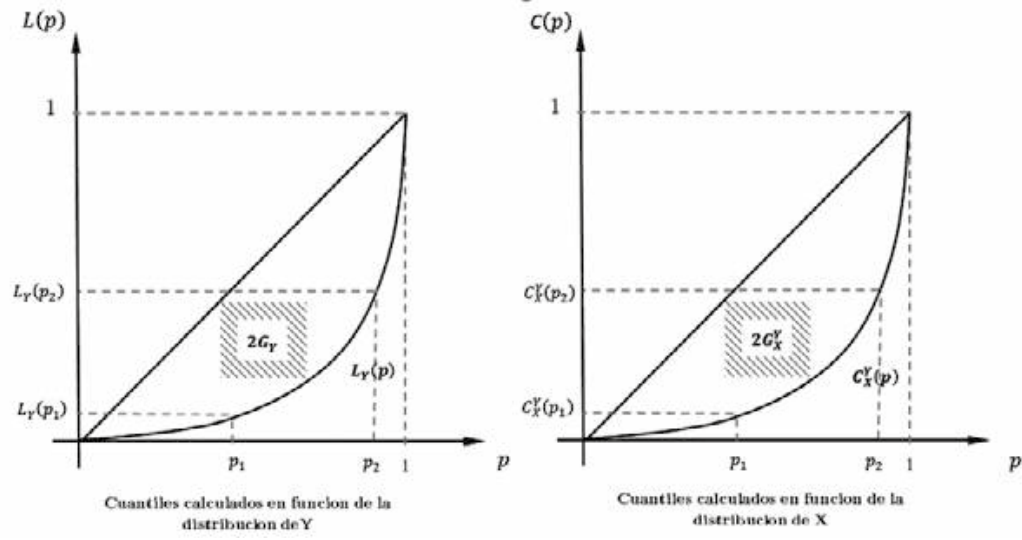

Fuente: Elaboración Propia

Un indicador bastante asociado con el espacio geométrico trazado por la curva de Lorenz, y cuya base sustenta la elaboración de otros índices de incidencia distributiva, es el Coeficiente de Gini $G_{Y}$. Este coeficiente mide las disparidades que posee la distribución de la variable $Y$ frente a una distribución uniforme, mediante dos veces el área que separa la curva de Lorenz $L_{Y}\left(p_{P}\right)$ y la recta de equidistribución. En el caso de una curva de concentración $C_{X}^{Y}(p)$, este índice se conoce como coeficiente de Cuasi-Gini $G_{X}^{Y}$.

Entre menor (mayor) sea el valor del coeficiente de Gini $G_{Y}$, mas (menos) equitativa será la distribución de $Y$; en otras palabras, la variable $Y$ se distribuye de manera más (menos) proporcional sobre todos sus cuantiles. La misma interpretación tiene el coeficiente de Cuasi-Gini $G_{X}^{Y}$, con la diferencia que la dispersión de la distribución de $Y$ se mide en términos de los cuantiles de la variable $X$. Duclos (2002) suministra una explicación detallada de estos y otros índices utilizados para medir la desigualdad.

\subsection{Análisis marginal del coeficiente de Gini por fuente de ingreso}

Los trabajos realizados por Lerman y Yitzaki (1985) constituyen los primeros sustentos metodológicos del análisis marginal de la desigualdad económica. Según estos autores, la elasticidad de Gini por fuente de ingreso $j$ (es decir, 
la variación relativa del coeficiente de Gini, respecto a un cambio marginal porcentual de la fuente de ingreso $j$ ) es:

$$
\frac{\partial G_{Y} / \partial e}{G_{Y}}=\frac{S_{j} G_{j} R_{j}}{G_{Y}}-S_{j}
$$

donde:

- $\quad R_{j}$ es el coeficiente de correlación de Gini entre la fuente de ingreso $j$ y el ingreso total $Y^{5}$.

- $G_{j}$ es el coeficiente de Cuasi-Gini de la fuente de ingreso $j$.

- $S_{j}$ es la proporción de la fuente de ingreso $j$ respecto al ingreso total $Y$.

- $\quad e$ es el cambio que experimenta la fuente de ingreso $j$, tal que $Y_{j}(e)=(1+e) Y_{j}$

De acuerdo con Stark (1986), esta elasticidad permite examinar el comportamiento la desigualdad económica a través de tres componentes: la importancia relativa de cada fuente de ingreso en el ingreso total $S_{j}$, la concentración de cada fuente $G_{j}$, y el grado de correlación de la fuente con el ingreso del hogar $R_{j}$.

De esta manera, se puede conocer cuando el cambio de una fuente de ingreso $j$ aumenta o disminuye la desigualdad del ingreso bruto. Por ejemplo, si la fuente ingreso posee una correlación positiva con el ingreso total $\left(R_{j}>0\right)$ y una concentración mayor que la desigualdad observada en el ingreso total $\left(G_{j}>G_{Y}\right)$, entonces la elasticidad de la desigualdad es positiva $\left(\frac{\partial G_{Y} / \partial e}{G_{Y}}>0\right)$.

Esta metodología ha sido extendida de varias formas. Yitzhaki (1990), Olkin y Yitzhaki (1992) y Garner (1993) son algunos casos que dan lugar a nuevas elasticidades y usos.

\subsection{Fuente de Información}

El presente estudio utilizóla Encuesta de Condiciones deVida(ECV)2013-2014, realizada por el Instituto Nacional de Estadísticasy Censos (INEC), paraanalizar la desigualdad económica en Ecuador. Esta encuesta recoge información de

5 De acuerdo a Stark et. al. (1986), la correlación de Gini cumple con las siguientes propiedades:

- Si $R_{j}=1\left(R_{j}=-1\right)$, entonces $Y_{i}$ es una función creciente (decreciente) de $Y$. Si $R_{j}=0$, entonces no existe función (lineal o no lineal) que relacione ambas variables. En este sentido, la correlación de Gini es muy similar a la correlación de Spearman.

- Si $Y_{j} Y$ son variables normalmente distribuidas, entonces $R_{j}$ coincide con la correlación de Pearson. 
109,694 individuos divididos en 28,970 hogares, que en términos poblacionales equivalen a 15,952,441 individuos y 4,346,025 hogares, respectivamente.

Los ingresos tomados de esta encuesta se encuentran registrados en la séptima sección "Actividades Económicas" y en la décima sección "Gastos, Otros Ingresos y Equipamiento del Hogar".

Esta información fue agrupada en 3 componentes con la finalidad de facilitar el análisis.

- Ingresos por actividad: comprende los ingresos de asalariados, y ganancias de cuenta propia y patronos, antes de impuestos.

- Ingresos por capital: comprende los ingresos por retornos de capital financiero (intereses, bonos, acciones) y capital físico (arriendo), antes de impuestos.

- Ingresos por transferencias: comprende las transferencias del gobierno por concepto de Bono de Desarrollo Humano (BDH) y Bono de la Vivienda (BV), las remesas remitidas desde el exterior, becas privadas y públicas, entre otras.

El análisis marginal de estas fuentes de ingresos se realizó en términos per-cápita del hogar, utilizando la escala de equivalencia propuesta por la $\mathrm{OCDE}^{6}$. De esta manera, se asegura que el cálculo de los índices de desigualdad y correspondientes elasticidades tome en cuenta la estructura y composición del núcleo familiar.

\section{Resultados y discusión}

\subsection{Ingresos por actividad}

Las distribuciones de la renta salarial y las ganancias son similares a la distribución del ingreso total, dadas las diferencias poco significativas de las curvas de concentración y la curva de Lorenz (Figura 5).

6 En esta escala, el adulto mayor tiene un peso dentro de la composición del hogar igual a 1, las personas mayores a 15 años tienen un peso de 0.7 y las personas menores a 14 años tienen un peso de 0.5 . 
Figura 5. Curvas de Lorenz del Ingreso por actividad

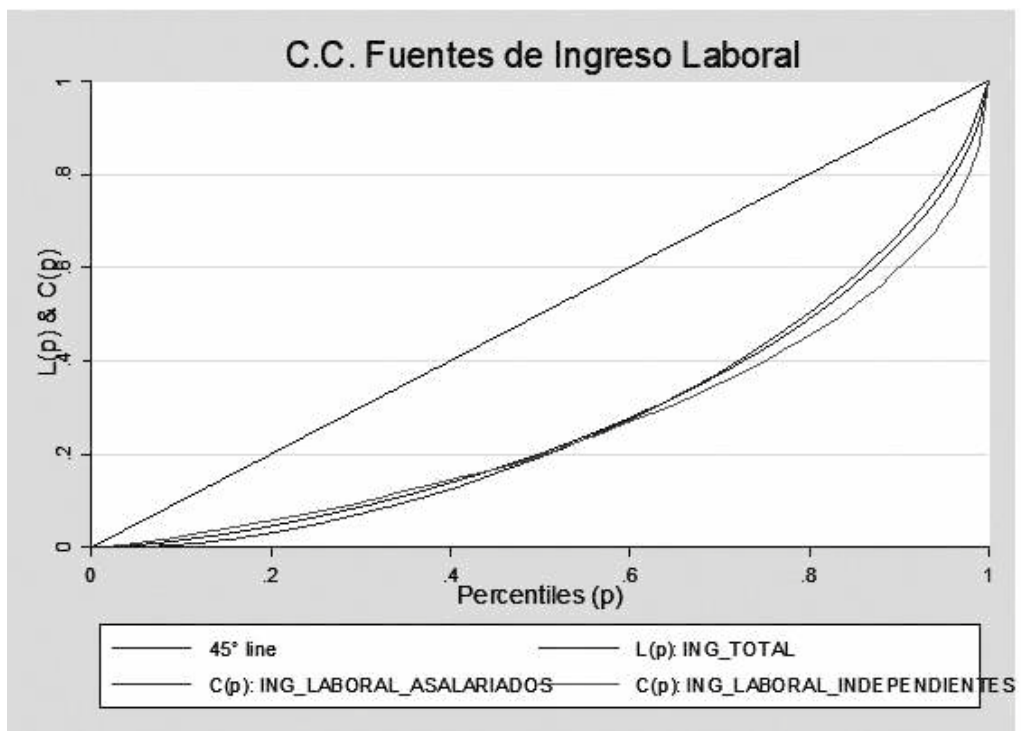

Fuente: Encuesta de Condiciones de Vida. Año 2013-2014

El coeficiente de Cuasi-Gini permite constatar esta situación (Tabla 2). Se observa que la dispersión de la renta laboral y el ingreso global son similares, y a la vez ligeramente inferiores a la dispersión presente en la renta patronal. Este resultado justifica en parte también la alta concentración de los ingresos de personas independientes para el $20 \%$ de la población más rica, la cual se ubica por encima de la renta que acumulan los asalariados, e incluso por encima del ingreso total.

Tabla 2. Distribución del Ingreso por actividad

\begin{tabular}{lccc}
\hline & Ingreso Total & $\begin{array}{c}\text { Ing. Laboral } \\
\text { Asalariados }\end{array}$ & $\begin{array}{c}\text { Ing. Laboral } \\
\text { Independientes }\end{array}$ \\
\hline $20 \%$ más pobres & $4.6 \%$ & $3.1 \%$ & $5.8 \%$ \\
$20 \%$ más ricos & $51.1 \%$ & $49.6 \%$ & $54.5 \%$ \\
Cuasi-Gini & 0.457 & 0.459 & 0.480 \\
\hline
\end{tabular}

Fuente: Encuesta de Condiciones de Vida. Año 2013-2014 


\subsection{Ingresos por retornos de capital}

La distribución del ingreso por retornos de capital (principalmente de origen financiero) es menos equitativa que la distribución del ingreso global, dado al gran distanciamiento que se produce entre las curvas de concentración y la curva de Lorenz del ingreso (Figura 6).

Figura 6. Curvas de Lorenz del Ingreso por retornos de capital.

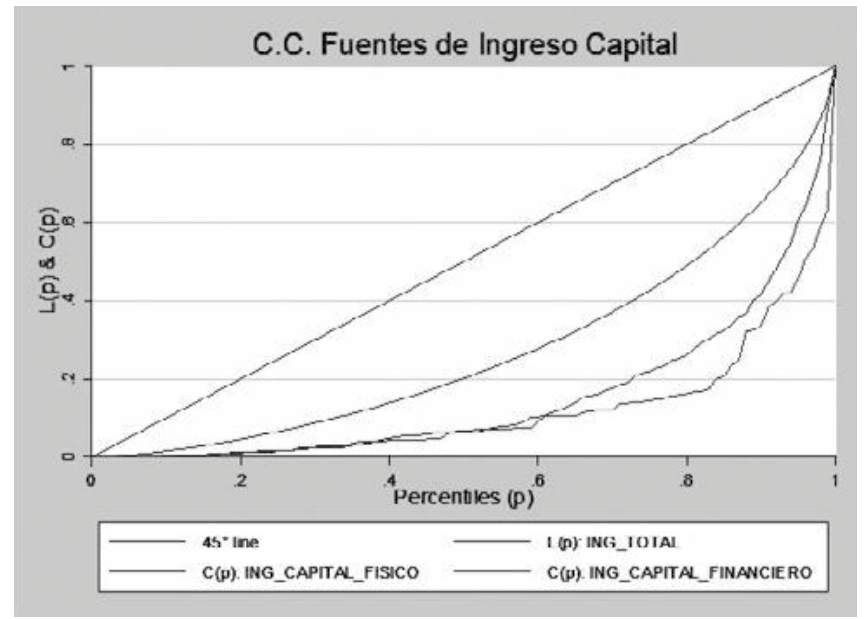

Fuente: Encuesta de Condiciones de Vida. Año 2013-2014

Esta situación se refleja también en el coeficiente de Cuasi-Gini, ya que el mismo adquiere valores superiores al 0.6 (Tabla 3) como consecuencia de la alta concentración existente. En el caso del capital financiero, esta concentración es superior al $80 \%$ para el $20 \%$ de la población más rica, conduciendo a niveles altos de polarización en esta fuente de ingreso.

Tabla 3. Distribución del Ingreso por retornos de capital.

\begin{tabular}{lccc}
\hline & Ingreso Total & $\begin{array}{c}\text { Ing. Capital } \\
\text { físico }\end{array}$ & $\begin{array}{c}\text { Ing. Capital } \\
\text { financiero }\end{array}$ \\
\hline 20\%más pobres & $4.6 \%$ & $1.1 \%$ & $1.3 \%$ \\
$20 \%$ más ricos & $51.1 \%$ & $73.9 \%$ & $83.8 \%$ \\
Cuasi-Gini & 0.457 & 0.698 & 0.767 \\
\hline
\end{tabular}

Fuente: Encuesta de Condiciones de Vida. Año 2013-2014 


\subsection{Ingresos por transferencias}

El ingreso por concepto de trasferencias tiene distintos patrones distributivos con respecto al ingreso total. En particular, transferencias como el Bono de Desarrollo Humano, el Bono de la Vivienda y las remesas son progresivas, ya que sus respectivas curvas de concentración se encuentran por encima de la curva de Lorenz. En otras palabras, para deciles de ingreso bajo, estas transferencias se acumulan en un mayor porcentaje al visto en la renta total. Por otro lado, las becas escolares y otras transferencias son regresivas, pues sus curvas de concentración al contrario se ubican por debajo de la curva de Lorenz del ingreso. (Figura 7).

Figura 7. Curvas de Lorenz del Ingreso por transferencias.

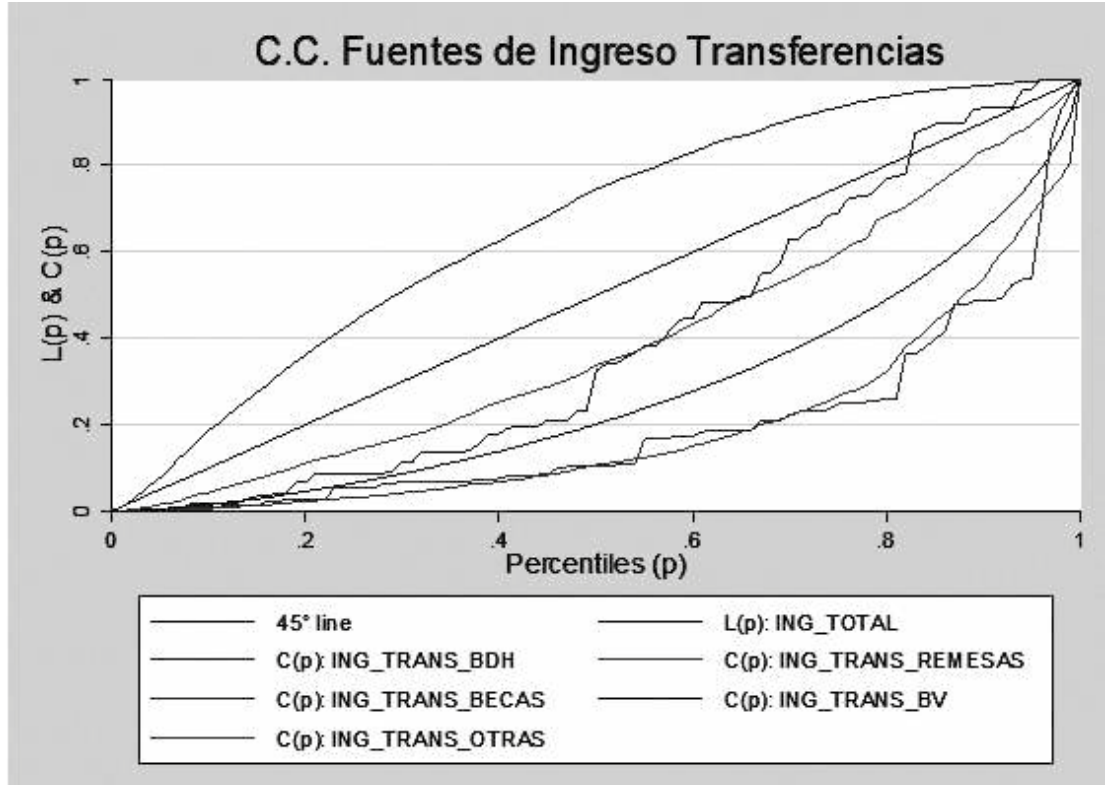

Fuente: Encuesta de Condiciones de Vida. Año 2013-2014

Estos rasgos distributivos se pueden corroborar mediante los coeficientes de Gini, tanto para las transferencias progresivas (Cuasi-Gini menores al Gini del Ingreso Bruto) como para las trasferencias regresivas (Cuasi-Gini mayores al Gini del Ingreso Bruto) (Tabla 4). 
Tabla 4. Distribución del Ingreso por transferencias.

\begin{tabular}{lccccc}
\hline & $\begin{array}{c}\text { Ingreso } \\
\text { Total }\end{array}$ & $\begin{array}{c}\text { Ing. trans. } \\
\mathrm{BDH}\end{array}$ & $\begin{array}{c}\text { Ing. trans. } \\
\text { Remesas }\end{array}$ & $\begin{array}{c}\text { Ing. trans. } \\
\text { Becas }\end{array}$ & $\begin{array}{c}\text { Ing. trans. } \\
\text { BV }\end{array}$ \\
\hline 20\%más pobres & $4.6 \%$ & $36.2 \%$ & $11.1 \%$ & $2.7 \%$ & $6.7 \%$ \\
20\%más ricos & $51.1 \%$ & $4.1 \%$ & $31.4 \%$ & $74 \%$ & $22.9 \%$ \\
Cuasi-Gini & 0.457 & -0.323 & 0.227 & 0.623 & 0.219 \\
\hline
\end{tabular}

Fuente: Encuesta de Condiciones de Vida. Año 2013-2014

En términos generales, estos aspectos justifican los altos niveles de concentración del Bono de Desarrollo humano, el Bono de la Vivienda y las remesas en más del $6 \%$ de la renta global para el $20 \%$ de la población más pobre (llegando hasta el $36.2 \%$ en el caso del BDH); así como los altos niveles de concentración de las becas, que alcanzan el $74 \%$ de la renta total para el $20 \%$ de la población más rica.

\subsection{Elasticidad de Gini por fuente de ingreso}

El análisis de desigualdad realizado para cada uno de los componentes del ingreso bruto, no es suficiente si se desea conocer su impacto distributivo. Para ello, es necesario estimar la elasticidad de Gini, con el objeto de identificar las fuentes de ingreso que contribuyen positiva o negativamente en la desigualdad económica y determinar en qué medida lo hacen (Tabla 5).

Tabla 5. Elasticidad de Gini por fuente de ingreso.

\begin{tabular}{lcccc}
\hline & $\begin{array}{c}\text { Participación } \\
\text { Ingreso (\%) }\end{array}$ & $\begin{array}{c}\text { Correlación } \\
\text { Gini }\end{array}$ & $\begin{array}{c}\text { Índice } \\
\text { Gini }\end{array}$ & $\begin{array}{c}\text { Elasticidad } \\
\text { Gini (\%) }\end{array}$ \\
\hline Ing_Laboral Asalariados & $60.2 \%$ & 0.788 & 0.582 & 0.72 \\
Ing_Laboral Independientes & $27.5 \%$ & 0.613 & 0.783 & 0.358 \\
Ing_Capital Físico & $1.73 \%$ & 0.715 & 0.976 & 0.04 \\
Ing_Capital Financiero & $0.12 \%$ & 0.769 & 0.997 & 0.003 \\
Ing_Trans BDH & $1.7 \%$ & -0.43 & 0.751 & -0.043 \\
Ing_Trans Remesas & $3.2 \%$ & 0.253 & 0.899 & 0.003 \\
Ing_Trans Becas & $0.07 \%$ & 0.624 & 0.998 & 0.001 \\
Ing_Trans BV & $0.07 \%$ & 0.219 & 0.998 & 0.000 \\
Ing_Trans Otras & $5.32 \%$ & 0.655 & 0.951 & 0.105 \\
\hline
\end{tabular}

Fuente: Encuesta de Condiciones de Vida. Año 2013-2014 
En primer lugar, se observa que los efectos más fuertes sobre la desigualdad provienen de los ingresos por concepto de trabajo y ganancias. Aquí, un incremento del $1 \%$ en las rentas salariales y rentas patronales, conduce a un aumento en el coeficiente de Gini de $0.72 \%$ y $0.358 \%$, respectivamente. Esto deja entrever el alto nivel de incidencia de la renta salarial y las ganancias sobre el ingreso total, como producto principalmente de su alta participación.

Con respecto a los retornos provenientes del capital, existe una pequeña contribución en la variación de la desigualdad, pese a la fuerte concentración de estas rentas y su alta correlación con el ingreso bruto (especialmente aquellas procedentes de capital financiero).

En el caso de las transferencias, sean públicas, privadas o provenientes del exterior, se pueden distinguir resultados más interesantes. Si bien, las elasticidades son menores en términos absolutos al $0.05 \%$, el Bono de Desarrollo Humano tiene una contribución negativa sobre la desigualdad del ingreso total. Es decir, un aumento de este subsidio ayudaría a reducir la desigualdad del ingreso, aunque de manera poco significativa. Este resultado es razonable debido al tipo de focalización que tiene este bono sobre el $40 \%$ de la población más pobre.

Por otro lado, las remesas, pese a ser progresivas de acuerdo al análisis realizado en secciones anteriores, contribuyen positivamente a la desigualdad total. Este resultado se debe principalmente a la relación proporcional que existe con el ingreso bruto; situación que también se replica para el Bono de la Vivienda.

\section{Conclusiones}

Durante los últimos quince años, Ecuador ha sostenido un crecimiento económico con avances en la distribución del ingreso, aunque poco significativas, lo cual podría evidenciar que la economía posiblemente se encuentra en la cima de la "u invertida" propuesta por Kuznets, donde puede ser estructuralmente difícil reducir la desigualdad económica. En este sentido, es imprescindible analizar la distribución de las distintas clases de ingresos y buscar alternativas efectivas de mejora.

El análisis realizado en el presente documento identifica las principales fuentes de ingresos que contribuyen a la desigualdad económica en Ecuador según datos la Encuesta de Condiciones de Vida 2013-2014, utilizando curvas de concentración y curvas de Lorenz, en conjunto con la metodología planteada por Lerman y Yitzaki (1985). Asimismo, suministra algunas pautas para la elaboración 
de políticas públicas que ayuden a disminuir de forma más pronunciada la desigualdad del ingreso, en línea con los principios de desarrollo instituidos en la constitución y el Plan Nacional del Buen Vivir.

En términos generales, este análisis permite entrever que:

- La desigualdad económica en Ecuador depende fuertemente de la distribución del ingreso de los asalariados. Varias pautas de política que pueden servir para disminuir la desigualdad del ingreso laboral, y con ello reducir efectivamente la desigualdad de la renta total, representan por ejemplo: incrementar el salario mínimo, garantizar los beneficios de ley, cerrar brechas socioeconómicas y de géneros, regular las escalas salariales en entidades públicas y privadas, dar mayor flexibilidad laboral, equiparar la remuneración por la naturaleza del trabajo, entre otros.

- Los ingresos por actividad independiente constituyen otro factor importante en la desigualdad económica. Pese a que su contribución sea inferior a la vista para ingresos laborales, su concentración en los percentiles de menor renta es mayor. Esta evidencia señala la necesidad de una política tributaria con un impuesto mayormente progresivo, deducciones focalizadas en MYPYMES y mejores estrategias de control que disuadan el fraude tributario, a fin incrementar el tipo impositivo sobre los ingresos percibidos por personas patronos y cuenta provistas, y mejorar la distribución del ingreso.

- La concentración de los retornos de capital es alta, sobre todo cuando se trata de capital financiero. No obstante, una política dirigida a regular las operaciones en este mercado, pese a las complicaciones por la alta informalidad, daría resultados poco significativos en la reducción de la desigualdad.

- El Bono de Desarrollo Humano, además de ser progresivo, ayuda a disminuir la desigualdad del ingreso, por lo que sería recomendable dar un diseño más controlado y eficaz, así como analizar las posibilidades para incrementar su cobertura y financiamiento.

- Las remesas recibidas desde el exterior, pese a ser progresivas, contribuyen positivamente a la desigualdad del ingreso. Esto señala la importancia de generar fuentes de trabajo bien remunerado (con todos los beneficios y seguridades salariales) que desincentiven la emigración al exterior. Esto comprende entre varias otras cosas, dar acceso a una educación diversificada y de alto nivel, a fin de equiparar las oportunidades de los trabajadores en cuanto a las actividades que desean desempeñar. 
- El Bono de la Vivienda y las Becas Escolares son transferencias que aumentan la desigualdad del ingreso, es decir que benefician mayormente a hogares de alto ingreso. Este hecho obliga la búsqueda e implementación de un diseño focalizado que permita dirigir estas transferencias a los estratos de menores recursos; más aun en el caso de las becas escolares, las cuales muestran un comportamiento regresivo. 


\section{Referencias}

- Alonso, José, (2008). En defensa de la teoría del desarrollo". Instituto Complutense de Estudios Internacionales. Cuadernos Económicos No. 78.

- Asamblea Nacional Constituyente.(2008). "Constitución de la República del Ecuador".

- Atkinson, Anthony (1970). "On the mesurement of inequality". Journal of Economic Theory. pp. 244-263.

- Coral del Rio, Carlos (2001). "Desigualdad, Polarizacion y Pobreza en la Distribución de la Renta en Galicia". Instituto de Estudios Económicos de Galicia. Universidad de Vigo

- Dikhanov, Yuri (2005). "Trends in Global Income Distribution, 1970-2000, and Scenarios for 2015". Human Development Report Office. World Bank.

- Duclos, Jean-Yves (2002). "Poverty and Inequity: Theory and Estimation". Déparment d'économique and CRÉFA. Université Laval, Canada.

- Garner, Thesia. (1993). "Consumer Expenditures and Inequality: An Analysis Based on Decomposition of the Gini Coefficient". Review of Economics and Statistics, 75(1):134-38.

- Hettne, Björn (1995), "Dimensions of "Another Development". En B. Hettne, Development Theory and the Three Worlds. Londres: Longman, Segunda edición, pp. 160-206.

- Kuznets, Simon (1953). "Shapes of Upper Income Groups in Income and Savings". University of Pennsylvania. National Bureau of Economic Research.

- Milanovic, Branko (2011). "A short history of global inequality: The past two centuries". Explorations in Economic History 48 (2011) 494-506.

- Núñez, José. (2006). "La desigualdad económica medida a través de las curvas de Lorenz". Revista de Métodos Cuantitativos para la Economía. Vol. 2, pp. 65-108.

- Lerman, Robert y Yitzhaki, Shlomo. (1985), "Income Inequality effects by income source: a new approach and application to the U.S". Review of Economics and Statistics. Vol 67 (1). pp. 151-156.

- Olkin, Ingram y Yitzhaki Shlomo (1992). "Gini Regression Analysis". International Statistical Review 10(2):185-196.

- Ponce, Juan; Vos, Rob (2014). "Redistribution without Structural Change in 
Ecuador: Rising and Falling Income Inequality in the 1990s and 2000". Libro : "Falling Inequality in Latin America: Policy Changes and Lessons"

- Programa de las Naciones Unidas para el Desarrollo. (2002). "¿A que llamamos desarrollo?" "Capitulo 1. Importancia del Desarrollo Humano: Una Base Conceptual".

- Ramírez, René (2008). "Igualmente Pobres, desigualmente Ricos". Programa de las Naciones Unidades para el Desarrollo.

- Rawls, John (1971). "A Theory of justice". Harvard University Press.

- Stark, Oded; Taylor, J. Edward y Yitzhaki, Shlomo. (1986). "Remittances and Inequality". The Economic Journal. Vol 96, pp. 722-740.

- Satrustegui, Koldo (2009). "Desarrollo, Subdesarrollo, Maldesarrollo y Postdesarrollo. Una Mirada Transdisciplinar sobre el Debate y sus Implicaciones". Carta Latinoamericana. Contribuciones en Desarrollo y Sociedad en América Latina.

- Sen, Amartya (1979). "Equality of What?". The Tanner Lecture on Human Values. Stanford University, pp. 195-220.

- Sen, Amartya, Foster, J. (2005). "On Economic Inequality". Clarendon Press. Segunda Edición.

- Pikety, Thomas (2014). "El capital en el siglo XXI". Cambridge, Massachusetts

- Veltmeyer, Henry (2001), 'The quest for another development'. En H. Veltmeyer y Anthony O'Malley, eds., Transcending Neoliberalism: Community-Based Development in Latin America, Bloomfield (CT): Kumarian Press, pp. 1-34.

- Wodon, Quentin (2000), Poverty and Policy in Latin America and the Caribbean, World Bank Technical. Paper No. 467:138, Washington.

- Wodon, Quentin; y Yitzhaki, Shlomo. (2002a). "Desigualdad y Bienestar Social, Banco Mundial, Washington"

- Wodon, Quentin; y Yitzhaki, Shlomo. (2002b). "Evaluating the Impact of Government Programs on Social Welfare: The Role of Targeting and the Allocation Rules among Programs Beneficiaries". Public Finance Review, Vol. 30, $\mathrm{N}^{\circ}$ 2:102-123.

- Yitzhaki Shlomo (1990). "On the Progressivity of Commodity Taxation". Working Paper $\mathrm{N}^{\circ} 187$, Department of Economics, Hebrew University, Jerusalem, Israel. 